angestellten Versuche ergaben, dass das Licht des Mondes zwei Tage vor dem letzten Viertel ein helles Spektrum mit deutlich wahrnehmbaren Fraunhofer'schen Linien giebt; zur Zeit der partialen Mondfinsterniss am 3. August 1887 lies ich (in Odessa) das Licht des Mondes auf den Spalt des Instruments fallen und wăhrend der ganzen Dauer der Finsterniss sah ich die Fraunhofer'schen Linien sehr deutlich, ohne etwas am Heliostat zu ändern. Die Linien waren so deutlich, dass Personen, die das Spektrum zum ersten Male sahen, dieselben auf den ersten Blick wahrnahmen. Was das Zöllner'sche Sternspektroskop anbetrifft, so hat es einen Integrationswinkel von $6^{\circ}$. Mit diesem Spektroskop konnte ich ebenfalls die Fraunhofer'schen Linien in den oben beschriebenen Fällen sehen, wenn auch nicht so deutlich wie im Spektroskop von Dubosq.

Das Wetter an den Tagen vor der Finsterniss gab wenig Hoffnung auf das Gelingen der Beobachtungen, so dass wir glaubten, dass wir luberhaupt nichts von der Finsterniss sehen würden. Am Tage der Finsterniss selbst war der Himmel von früh an mit dichten Wolken tuberzogen, aber gegen Anfang derselben fingen die Wolken an lockerer zu werden. Vor der totalen Phase kam die Sonne zwischen grossen Cumulus-Wolken einige Male zum Vorschein, aber auf eine so kurze Zeit, dass ich die zwei Spiegel des Heliostats bis auf eine Viertelstunde vor der Totalität nicht aufstellen konnte. Ich entschloss mich daher, mit dem etwas lichtschwächeren Spektroskop von Zöllner zu beobachten und das Auge möglichst im Dunkel zu halten. $I^{3} / 4$ Minute vor der Totalität kam die Sonnensichel wieder durch Wolken zum Vorschein und ich benutzte diese Gelegenheit, um mit dem Zöllner'schen Spektroskop nach ihr zu sehen; obgleich ich das Spektroskop nur auf einige Secunden auf dieselbe richtete, erblickte ich deutlich die Fraunhofer'schen Linien. Der Anfang der Totalität war durch die Wolken nicht sichtbar, einige Secunden nachher kam aber die Sonne zwischen Wolken zum Vorschein und man konnte bald durch lockere, bald durch dichtere, die Sonne fast zum Verschwinden verdeckende Wolken die Corona erkennen. Trotz aller Anstrengung des Auges konnte ich im Spektrun derselben die Fraunhofer'schen Linien nicht sehen, auch die hellen Linien wurden nicht sichtbar. Das Spektrum erschien mir continuirlich und ziemlich hell, etwa so hell wie das des Vollmondes. Als die Sonne während der totalen Phase mit dichteren Wolken bedeckt wurde, wandte ich mich zu meinem Notizbuche, um die Beobachtungen aufzuschreiben; durch die grosse Helligkeit des Coronalichtes betroffen, stellte ich die Lampe, die mein Notizbuch erhellte, fort und konnte ohne kunstliche Beleuchtung bequem schreiben und die Uhr ablesen.
Während der totalen Phase waren der Himmel und die Wolken am südlichen und östlichen Theile des Himmels stark gefärbt erleuchtet; besonders auffallend war ein breiter dem Horizonte parallel gelegener Streifen von eigenthümlich grünlich-gelber Farbe. Obgleich zu Anfang der Totalität die Sonne nicht sichtbar war, so war doch der Unterschied in der Beleuchtung vor und nach der Totalität so gross, dass man den Anfang derselben, auch ohne die Sonne anzusehen, erkennen konnte. Windstoss oder Windstille während der 'Totalität war nicht zu bemerken, auch keine fühlbare Temperaturabnahme habe ich bemerkt. Meine Frau, die diese Finsterniss in Petrowsk betrachtete, war von mir beauftragt, auf die beweglichen Schatten beim Anfang und Ende der totalen Phase, sowie auch auf den Schatten eines senkrecht auf weisses Papier gestellten Bleistifts Acht zu geben. Die beweglichen Schatten waren nicht zu beobachten, aber der Bleistift warf zwei Schatten, einen von der Seite der Corona und den anderen von dem südöstlichen Theile des Horizonts. Nach dem Ende der totalen Phase kam die Sonne aus den Wolken hervor und schien auf klarem Himmel fast eine Stunde lang; gegen die Zeit des letzten Contactes verschwand sie jedoch wiederum zwischen Wolken, so dass derselbe nicht beobachtet werden konnte.

In Odessa beobachtete die Finsterniss Herr Zwietinowitsch am $61 / 2$ zöll. Refractor von Cooke. Der Anfang derselben konnte wegen der Wolken nicht gesehen werden; der letzte Contact wurde beobachtet $2 \mathrm{u} \mathrm{Ig}^{\mathrm{h}} 4^{\mathrm{m}} 2^{\mathrm{s}} \cdot 49$ M.Z. Odessa.

Schreiben des Herrn Dr. $N$. Herz, Leiter der v. Kuffner' schen Sternwarte in Ottakring bei Wien, vom r. October.

Die grösste Phase war nicht zu beobachten, da die Sonne erst nachher aus den schweren Wolken, die den Osthimmel bedeckt hatten, herauskam. Der letzte Contact war sehr gut zu erhalten, da der Hinmel völlig rein geworden war; selbst der gewöhnlich über Wien lagernde Dunst, der bei uns den Beobachtungen auf der Ostseite oft sehr hinderlich ist, war ausnahmsweise ganz zerstreut, so dass man die Conturen der etwa 60 bis 80 Kilometer entfernten kleinen Karpathen völlig klar sah.

Der Contact wurde beobachtet August $181^{\mathrm{h}} 6^{\mathrm{m}} 10^{\mathrm{s}}$ M. Z. Wien, Sternwarte Ottakring. Die geographischen Coordinaten der Sternwarte sind: $\lambda=0^{\mathrm{h}} \mathrm{I}^{\mathrm{m}} 3^{\mathrm{s}}$ östlich Berlin, $\varphi=+48^{\circ} 12^{\prime} 4^{\prime \prime}$.

Beobachtung auf der Sternwarte in Lund. (Aus einem Schreiben von Dr. Dunér vom 22. October.)

Ende der Finsterniss $18^{\mathrm{h}} 2^{\mathrm{m}} 5^{1 !} \cdot 5$ M. Z. I und.

Beob. Dunér am Fernrohre von Merz, 34 Lin. Oeffnung, 8o malige Vergrösserung.

\title{
Beobachtung des Planeten (270) (Peters Oct. 8).
}

\author{
1887 Oct. $131^{\text {h }} 3^{1^{\mathrm{m}} 22^{3}}$ M. Z. Dusseldorf $\Delta \alpha=-5^{\mathrm{m}} 39^{\mathrm{g}} 3^{2}, \Delta \delta=+2^{\prime} 53^{\prime \prime} 4$, Vgl. 6 \\ $\alpha$ app. $=1^{\mathrm{h}_{1} 2^{\mathrm{m}} 24^{3}: 82}(8.309 \mathrm{n}), \delta$ app. $=+11^{\circ} 5^{\prime \prime} 20^{\prime \prime} 8(0.746)$. \\ Vergleichstern (1887.0): $\alpha=I^{\text {h }} 18^{m_{1} !_{12}}+3^{5} .02, \delta=+11^{\circ} 49^{\prime} 12^{\prime \prime 0}+15^{\prime \prime} 4 \operatorname{Lam}_{4} 198$. \\ $R$. Luther.
}

Düsseldorf 1887 Oct. 15 . 